\title{
On the Connection between Phase Shifts and Scattering Potential
}

\author{
V. BARgMANN \\ Princeton University, Princeton, New Jersey
}

\begin{abstract}
Construction and discussion of phase equivalent potentials, i.e., of potentials which give equal phase shifts for $S$-waves. In particular, it is shown that the phase shifts do not determine the energy of bound states, and that there exist continuous families of phase equivalent potentials.
\end{abstract}

$T$ HE elastic scattering cross section of a particle of energy $E$ moving in a central field of force is determined by the phase shifts $\eta_{l}(k)$ of the partial waves (of different angular momenta $l$ ) into which the particle's Schrödinger function may be decomposed. Here, $k$ is the wave number, and, in appropriate units, $E=k^{2}$. (We restrict ourselves to the non-relativistic case.) One may ask to what extent the scattering potential $V(r)$ is determined (a) by the function $\eta_{l}(k)$ for a fixed angular momentum (for example, $l=0$ ), (b) by all functions $\eta_{l}(k)(l=0,1,2, \cdots)$. The answer to the second question follows from a recent investigation by $N$. Levinson. ${ }^{1}$ Two potentials which are known to decrease rapidly enough at large distances from the scattering center are identical if they give the same phase shifts for all $l$. (See Sec. 6 below.) The present paper deals mainly with the first question, specifically, with the case $l=0$ ( $S$-scattering).

Our method consists in studying "phase equivalent" potentials, i.e., potentials with the same function $\eta(k)$. (In what follows, $\eta(k)$ stands for $\eta_{0}(k)$.) An example of two phase equivalent potentials has recently been published. ${ }^{2}$ In the present paper we shall construct additional examples in a somewhat more systematic fashion. In each case the Schrödinger equation may be solved in terms of elementary functions, so that the phase shift $\eta(k)$ may be explicitly computed. N. Levinson has proved $^{3}$ that two phase equivalent potentials are identical if both decrease rapidly enough and if neither gives rise to a bound state. It has not been possible to formulate any other reasonable qualitative criteria for phase equivalence. In fact, phase equivalent potentials may have a markedly different appearance. They may have bound states of different energy; there even exist continuous families of phase equivalent potentials.

Connection with the theory of the S-matrix. A spherically symmetric solution of the Schrödinger equation has, for large distances, the asymptotic form $(c / r)\left(S(k) e^{i k r}\right.$ $\left.-e^{-i k r}\right)$ where $c$ is a constant and $S(k)=e^{2 i \eta(k)}$. The

${ }^{1}$ N. Levinson, Phys. Rev. 75, 1445 (1949). I am greatly indebted to Professor Levinson for communicating his results prior to publication.

Note added in proof: Professor E. A. Hylleraas has kindly informed me that he has independently arrived at Levinson's main conclusions.

2 V. Bargmann, Phys. Rev. 75, 301 (1949).

${ }^{3}$ In a paper to be published soon. amplitude of the scattered wave is $\sim(S(k)-1) / 2 i k r$, and $S(k)$ is a proper value of the $S$-matrix. By analytic continuation, the function $S(k)$ may be defined for complex $k$, and, in particular, for imaginary values $k=-i_{\kappa}(\kappa>0)$. As H. A. Kramers ${ }^{4}$ observed, the stationary states should then be obtained from the zeros of $S(k)$ (for $k=-i \kappa$ ). S. T. $\mathrm{Ma},{ }^{5}$ however, discovered the existence of "redundant" zeros of $S(k)$, i.e., of zeros which do not correspond to bound states, and R. Jost ${ }^{6}$ showed in a careful investigation (on which the present paper is based) that the redundant zeros are connected with singularities of the function $f(k, r)$ (see Sec. 1 below). The existence of phase equivalent potentials with different bound states shows that, in general, the knowledge of $S(k)$ is certainly insufficient for the determination of the bound states, irrespective of any further conditions which may be added to the equation $S(k)=0$. One might either rule out the redundant zeros by requiring the potentials to decrease faster than any exponential function (see Sec. 6) -which seems hardly justifiable - or else one would have to use the totality of all $S_{l}(k)$ (for all angular momenta) in order to determine the bound states. The latter alternative, however, would probably lead to extreme difficulties if one attempted to treat problems more complicated than the motion of a particle in a central field of force.

\section{INTRODUCTORY REMARKS}

The functions $f(k, r)$

Let $\psi(r)$ be the wave function of the particle for angular momentum zero. Then

$$
\phi^{\prime \prime}+k^{2} \phi=V(r) \phi
$$

where $\phi=r \cdot \psi$. (The energy $E$ equals $k^{2}$.) We specifically assume that $\int_{0}^{\infty}|V(r)| d r$ is finite, so that the scattering theory may be applied. Following Jost $^{6}$ we introduce two independent solutions $f(k, r)$ and $f(-k, r)$ of (1.1) which are defined by their asymptotic behavior for large $r$, viz., $\lim _{r \rightarrow \infty} e^{i k r} f(k, r)=1$, and $\lim _{r \rightarrow \infty} e^{-i k r} f(-k, r)=1$. Moreover, we set

$$
f(k)=f(k, 0) ; \quad f(-k)=f(-k, 0) .
$$

${ }^{4}$ Quoted by C. M $\phi$ ller, Kgl. Danske Vid. Sels. Math.-Fys. Medd. 24, No. 19 (1946), see p. 3.

${ }_{5}^{5}$ S. T. Ma, Phys. Rev. 69, 668 (1946); 71, 195 (1947). See also

D. ter Haar, Physica 12, 509 (1946).

${ }^{6}$ R. Jost, Helv. Phys. Acta 20, 256 (1947). 
For real non-vanishing $k, f(k, r)$ and $f(-k, r)$ are complex conjugate, continuous in $k$ and $r, f(k) \neq 0$, and $\lim _{k \rightarrow \infty} f(k)=1$. In case (1.8) holds, $f(k, r)$ is also continuous at $k=0$. The function

$$
\phi=(1 / 2 i|f(k)|)\{f(k) f(-k, r)-f(-k) f(k, r)\}
$$

vanishes at the origin $(r=0)$, and is therefore, apart from a constant factor, the only admissible solution of (1.1) for a given wave number $k$. Asymptotically,

$$
\phi \sim \sin (k r+\eta(k))
$$

where the phase shift $\eta(k)$ is determined by $e^{i \eta(k)}=f(k) /|f(k)|$ or $e^{2 i \eta(k)}=S(k)=f(k) / f(-k)$.

Since $f(k) \rightarrow 1$ as $k \rightarrow \infty$, we may set $\eta(\infty)=0$. Then the following definition may be shown to be equivalent to (1.5). Let, for a fixed $k, \rho_{n}(k)$ be the $n$th zero of $\phi$ (cf. (1.3)) on the interval $0<r<\infty$, and set

$$
\eta(k)=\lim _{n \rightarrow \infty}\left(n \pi-k \rho_{n}(k)\right) .
$$

The definition of $f(k, r)$ by its asymptotic behavior may be extended to complex $k$ with negative imaginary parts (see Jost ${ }^{6}$ ), and $f(k, r)$ is a regular analytic function in the lower half plane. By analytic continuation it may also be defined in the upper half plane, but will, in general, have singularities. Consider, in particular, an imaginary value of $k, k=-i_{\kappa}(\kappa>0)$. The corresponding energy $\left(E=k^{2}\right)$ is negative, and $E$ is a stationary energy value if and only if the exponentially decreasing solution $f(k, r)$ vanishes at the origin, i.e., if

$$
f(-i \kappa)=0, \quad\left[\kappa>0, E=-\kappa^{2}\right] .
$$

In addition, there may be a bound state of energy $E=0$ which is not determined by the equation $f(0)=0$. Such a bound state cannot occur, however, if for large $r, V(r)$ decreases rapidly enough, so that

$$
\int_{0}^{\infty} r|V(r)| d r<\infty
$$

(For a proof, see the appendix.) If (1.8) holds, we may therefore conclude that two potentials with the same $f(k)$ are phase equivalent and have the same bound states.

The phase shift $\eta(k)$ determines $S(k)$ [see (1.5)] but not $f(k)$. The vanishing of $S(k)$, on the other hand, does not imply the vanishing of $f(k)$, because it may be due to an infinity of $f(-k)$. For this reason, two phase equivalent potentials may have bound states of different energy values. The number $m$ of bound states, however, is determined by the phase shifts if $\int_{0}^{\infty} r^{2}|V(r)| d r<\infty$. Then $f(k)$ is a differentiable function of $k$ (including $k=0),{ }^{7}$ and it can be shown that

$$
\eta(0)=\left\{\begin{array}{ccc}
m \pi & \text { if } & f(o) \neq 0 \\
\left(m+\frac{1}{2}\right) \pi & \text { if } & f(o)=0 .
\end{array}\right.
$$

\footnotetext{
${ }^{7}$ I owe this remark to $\mathrm{N}$. Levinson.
}

\section{POTENTIALS OF LINEAR TYPE}

The potentials to be considered will be so chosen that the corresponding functions $f(k)$ are rational. Set $\phi=e^{-i k r} \chi$. From (1.1),

$$
\chi^{\prime \prime}-2 i k \chi^{\prime}=V(r) \chi .
$$

Let $\chi(k, r)$ be a family of solutions of (2.1) for which $\chi(k, \infty)=\lim _{r \rightarrow \infty} \chi(k, r)$ exists and is different from zero. Then

$$
f(k, r)=e^{-i k r} \frac{\chi(k, r)}{\chi(k, \infty)} ; \quad f(k)=\frac{\chi(k, o)}{\chi(k, \infty)} .
$$

We now impose the condition that the $\chi(k, r)$ be polynomials in $k$, and we shall construct the corresponding potentials $V(r)$. By (2.2), $f(k)$ will be rational, and it will be possible to find phase equivalent potentials.

Potentials of linear type. $\chi(k, r)$ is assumed to have the form

$$
\chi(k, r)=2 k+i a(r) .
$$

Inserting $\chi$ in (2.1), we find $V=a^{\prime}$, and $V a=a^{\prime \prime}$, or

$$
a^{\prime} a=a^{\prime \prime} ; \quad V=a^{\prime} \text {. }
$$

With $a_{\infty}=\lim _{r \rightarrow \infty} a(r)$, and $a_{0}=a(o)$, we obtain from (2.2)

$$
f(k, r)=e^{-i k r} \frac{2 k+i a(r)}{2 k+i a_{\infty}} ; \quad f(k)=\frac{2 k+i a_{0}}{2 k+i a_{\infty}} .
$$

It is easily shown that, for a non-vanishing potential $V$, the function $a(r)$ must be real. Integrating the first Eq. (2.4), we find

$$
a^{\prime}-\frac{1}{2} a^{2}=2 c \quad(c=\text { const. })
$$

To solve this equation it is convenient to introduce

$$
w(r)=c^{\prime} \cdot \exp \left(-\frac{1}{2} \int_{0}^{r} a\left(r^{\prime}\right) d r^{\prime}\right)
$$

where $c^{\prime}$ is an arbitrary positive constant, so that

$$
w(r)>0 \text { for all } r \text {. }
$$

FIG. 1. Two phase equivalent potentials of the form $V_{1}[\rho=2$ $\sigma=1]$ with parameters $\theta=0.5(-)$ and $\theta=5(---)$

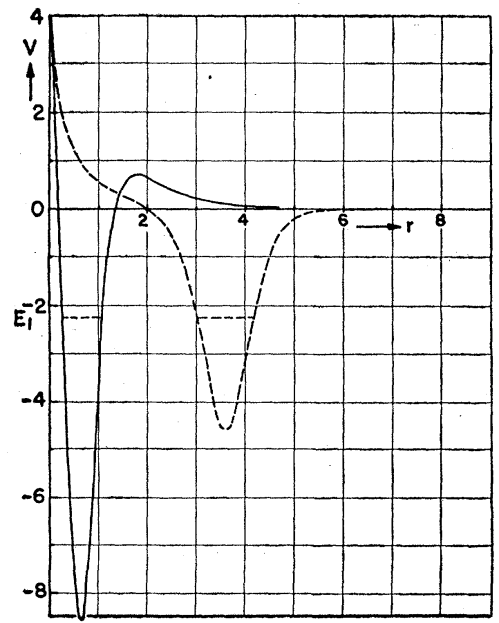




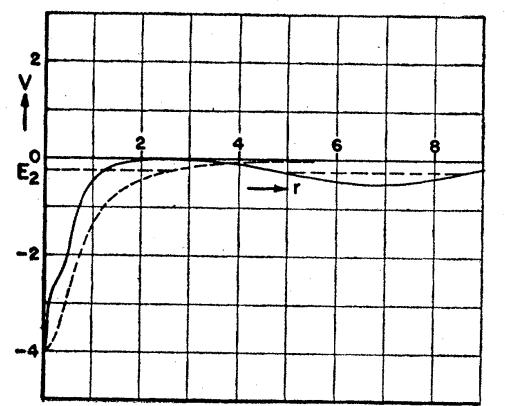

Fig. 2. Two potentials $V_{2}[\rho=2, \sigma=1]$ with parameters $\theta=\overrightarrow{3}$ $(-)$ and $\theta=0$ $(-\cdots)$. The potentials in Fig. 1 and Fig. 2 are phase equivalent.

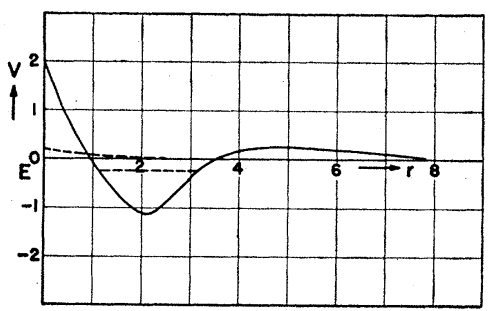

Fig. 3. Two phase equivalent potentials $V_{6} \quad[\alpha=1, \quad \gamma=2]$ $=0.949, \beta=-0.311]$ $(---)$.

Clearly, the value of $c^{\prime}$ is immaterial. It follows that

$$
a=-2 w^{\prime} / w ; \quad V=a^{\prime}=2\left(\left(w^{\prime} / w\right)^{2}-w^{\prime \prime} / w\right)
$$

Inserting this in (2.6) we finally obtain

$$
w^{\prime \prime}+c w=0 \text {. }
$$

Every solution of (2.10) which satisfies the inequality $w>0$ leads to a potential $V$ (see (2.9)) of linear type.

(1) $c=0$. In view of (2.8), and with an appropriate choice of $c^{\prime}, w(r)=r+\alpha(\alpha>0)$. Hence

$$
a(r)=-2 /(r+\alpha) ; \quad V(r)=2 /(r+\alpha)^{2} .
$$

(2) $c \neq 0$. The inequality $w>0$ implies that $c<0$, and we set $c=-\left(\frac{1}{2} \lambda\right)^{2}$. A solution which leads to a nonvanishing potential $V$ must have the form $w=e^{\frac{1}{2} \lambda r}$ $+\beta e^{-\frac{1}{2} \lambda r}(\beta>-1)$, so that

$$
a(r)=\lambda \frac{\beta e^{-\lambda r}-1}{\beta e^{-\lambda r}+1} ; \quad V(r)=-2 \beta \lambda^{2} \frac{e^{-\lambda r}}{\left(\beta e^{-\lambda r}+1\right)^{2}} .
$$

$V$ is an Eckart potential, ${ }^{8}$ and $a(r)$ equals the function $\mu(r)$ in reference 2 (see (7a)). In particular, by (2.5),

$$
f(k)=\frac{2 k+i \nu}{2 k-i \lambda} ; \quad \nu=a_{0}=\lambda \frac{\beta-1}{\beta+1} .
$$

\section{POTENTIALS OF QUADRATIC TYPE}

Here

$$
\chi(k, r)=4 k^{2}+2 i k a(r)+b(r)
$$

and

$$
\left\{\begin{array}{c}
f(k, r)=e^{-i k r} \frac{4 k^{2}+2 i k a(r)+b(r)}{4 k^{2}+2 i k a_{\infty}+b_{\infty}}, \\
f(k)=\frac{4 k^{2}+2 i k a_{0}+b_{0}}{4 k^{2}+2 i k a_{\infty}+b_{\infty}}
\end{array}\right.
$$

${ }^{8}$ C. Eckart, Phys. Rev. 35, 1303 (1930).
It follows from (2.1) that $V=a^{\prime}, V a=a^{\prime \prime}-b^{\prime}, V b=b^{\prime \prime}$. Hence

$$
L=b^{\prime}-a^{\prime \prime}+a^{\prime} a=0, \quad L_{1}=b^{\prime \prime}-a^{\prime} b=0 ; \quad V=a^{\prime} .
$$

We first observe that any potential of linear type may also be considered to be of quadratic type. In fact, if (2.1) admits a family of solutions $\chi(k, r)=2 k+i a(r)$, it also admits, of course, the family

$$
\begin{aligned}
\chi_{1}(k, r)=(2 k+i a(r)) & (2 k+i \tau) \\
= & 4 k^{2}+2 i k(a(r)+\tau)-\tau a(r),
\end{aligned}
$$

where $\tau$ may be any real or complex constant. A simple discussion of the Eqs. (3.3) shows that, apart from this trivial case, the functions $a(r)$ and $b(r)$ are real and are uniquely determined by $V(r)$.

To solve (3.3), we replace the set $L=0, L_{1}=0$, by the equivalent set $L=0, M=0$, where $M=b L+a L_{1}=b b^{\prime}$ $-b a^{\prime \prime}+a b^{\prime \prime}$. These equations have the two first integrals $\left(c_{1}, c_{2}\right.$ constant)

$$
\begin{aligned}
b-a^{\prime}+\frac{1}{2} a^{2} & =2 c_{1}, \\
\frac{1}{2} b^{2}-b a^{\prime}+a b^{\prime} & =2 c_{2} .
\end{aligned}
$$

Introducing again the function $w(r)[$ see $(2.7)]$, we find

$$
a=-2 w^{\prime} / w ; \quad V=a^{\prime}=2\left(\left(w^{\prime} / w\right)^{2}-w^{\prime \prime} / w\right)
$$

and, from (3.5),

$$
b=2\left(c_{1}-w^{\prime \prime} / w\right) .
$$

Inserting these expressions in (3.6) we finally obtain, after multiplication with $\frac{1}{4} w^{2}$,

$$
Q=w^{\prime} w^{\prime \prime \prime}-\frac{1}{2}\left(w^{\prime \prime}\right)^{2}-c_{1}\left(w^{\prime}\right)^{2}+\frac{1}{2}\left(c_{1}^{2}-c_{2}\right) w^{2}=0 .
$$

(3.7), (3.8), and (3.9) are equivalent to the original set of equations.

The derivative of $Q$ has the form

$$
\frac{d Q}{d r}=w^{\prime} R ; \quad R=w^{(4)}-2 c_{1} w^{\prime \prime}+\left(c_{1}^{2}-c_{2}\right) w .
$$

Since the solutions of (3.3) are necessarily analytic, and since $d Q / d r=0$, it follows that either $w^{\prime}=0$, or $R=0$. Discarding the trivial solution $w^{\prime}=0$ (which implies $V=0$ ) we have, therefore, the linear equation

$$
R=0 \text {. }
$$

Then, by (3.10), $Q=$ const., so that (3.9) reduces to an initial condition.

In general, the solutions of $R=0$ are linear combinations of exponential functions $e^{\omega r}$ whose exponents are obtained from $\omega^{4}-2 c_{1} \omega^{2}+\left(c_{1}^{2}-c_{2}\right)=0$, i.e.,

$$
\omega^{2}=c_{1} \pm\left(c_{2}\right)^{\frac{1}{2}} \text {. }
$$

If multiple roots occur (i.e., if $c_{2}$ or $c_{1}{ }^{2}-c_{2}$ or both vanish) the solutions of $R=0$ degenerate in the familiar way.

Without discussing the solutions of (3.9) in detail, we shall merely select some examples of phase equivalent 
potentials. The computations involved are elementary and straightforward. It will suffice to state the main results.

\section{EXPONENTIALLY DECREASING POTENTIALS}

(a) Assume that the four roots $\omega$ given by (3.12) are real and distinct, and denote them by

$$
\rho,-\rho, \sigma,-\sigma \quad(\rho>\sigma>0)
$$

so that

$$
c_{1}=\frac{1}{2}\left(\rho^{2}+\sigma^{2}\right), \quad c_{2}=\frac{1}{4}\left(\rho^{2}-\sigma^{2}\right)^{2} .
$$

The solutions of $R=0$ are

$$
w(r)=\left(\gamma_{1} e^{\rho r}+\gamma_{2} e^{-\rho r}\right)+\left(\delta_{1} e^{\sigma r}+\delta_{2} e^{-\sigma r}\right),
$$

and in view of the equation $Q=0$ the coefficients are subject to the condition

$$
\rho^{2} \gamma_{1} \gamma_{2}=\sigma^{2} \delta_{1} \delta_{2} .
$$

It is easily shown that $w(r)$ leads to a potential of linear type of the form (2.12) unless all coefficients $\gamma, \delta$ are different from zero. We set, therefore, $\gamma_{1}=\sigma, \gamma_{2}=\sigma \alpha \beta$, $\delta_{1}=\rho \alpha, \delta_{2}=\rho \beta$, so that

$$
\left\{\begin{aligned}
w(r) & =\sigma\left(e^{\rho r}+\alpha \beta e^{-\rho r}\right)+\rho\left(\alpha e^{\sigma r}+\beta e^{-\sigma r}\right), \\
w^{\prime}(r) & =\rho \sigma\left(e^{\rho r}-\alpha \beta e^{-\rho r}+\alpha e^{\sigma r}-\beta e^{-\sigma r}\right) .
\end{aligned}\right.
$$

Using (4.1) and (4.2), one finds from (3.7) and (3.8) that $a_{\infty}=-2 \rho, b_{\infty}=\sigma^{2}-\rho^{2}$, and

$$
4 k^{2}+2 i k a_{\infty}+b_{\infty}=[2 k-i(\rho-\sigma)][2 k-i(\rho+\sigma)] .
$$

Furthermore, for large $r$,

$$
\left\{\begin{array}{lll}
V(r) \sim-2(\rho / \sigma) \alpha(\rho-\sigma)^{2} e^{-(\rho-\sigma) r} & \text { if } & \alpha \neq 0 \\
V(r) \sim-2(\rho / \sigma) \beta(\rho+\sigma)^{2} e^{-(\rho+\sigma) r} & \text { if } & \alpha=0 .
\end{array}\right.
$$

For $r=0$,

$$
\left\{\begin{aligned}
a_{0} & =-2 \rho \sigma(1+\alpha)(1-\beta) / w(0) ; \\
b_{0} & =\left(\sigma^{2}-\rho^{2}\right)[\sigma(1+\alpha \beta)-\rho(\alpha+\beta)] / w(0), \\
w(0) & =\sigma(1+\alpha \beta)+\rho(\alpha+\beta) .
\end{aligned}\right.
$$

In what follows we restrict ourselves to those solutions for which $a_{0}=0$. Then, by (4.6), either $\alpha=-1$, or $\beta=1$.

(b) The case $\alpha=-1$. Here, the inequality $w>0$ holds for all $r$ if and only if $\beta>1$ (see (4.3)), and we may set $\beta=e^{2 \theta}(\theta>0)$. Then the corresponding potential, which we denote by $V_{1}(r)$, is found to be

$$
V_{1}(r)=\frac{\rho \sigma\left\{4 \rho \sigma+(\rho-\sigma)^{2} \cosh [(\rho+\sigma) r-2 \theta]-(\rho+\sigma)^{2} \cosh (\rho-\sigma) r\right\}}{\{\sigma \sinh (\rho r-\theta)-\rho \sinh (\sigma r-\theta)\}^{2}} .
$$

By (4.6), $b_{0}=(\rho+\sigma)^{2}$, and in view of (3.2) and (4.4),

$$
f_{1}(k)=\frac{2 k+i(\rho+\sigma)}{2 k-i(\rho-\sigma)} .
$$

Since $f_{1}(k)$ does not depend on $\beta$ (or $\theta$ ) any more, the $V_{1}(r)$ form a continuous family of phase equivalent potentials, all of which have a bound state of energy

$$
E_{1}=-\frac{1}{4}(\rho+\sigma)^{2}
$$

since $f_{1}(-i \kappa)=0$ if $\kappa=\frac{1}{2}(\rho+\sigma)$. All potentials have the same asymptotic form (see (4.5)), and the same value at the origin, viz., $V_{1}(0)=2 \rho \sigma$.

(c) The case $\beta=1$. Here the remaining parameter must be restricted to values $\alpha>-1$ in order that $w(r)$ be positive. According as $-1<\alpha<0, \alpha=0, \alpha>0$, the corresponding potentials may be written in the form

$$
\begin{aligned}
& V_{2}(r)=\frac{\rho \sigma\left\{4 \rho \sigma+(\rho-\sigma)^{2} \cosh (\rho+\sigma) r-(\rho+\sigma)^{2} \cosh [(\rho-\sigma) r+2 \theta]\right\}}{\{\sigma \sinh (\rho r+\theta)-\rho \sinh (\sigma r-\theta)\}^{2}}, \\
& V_{3}(r)=\frac{-2(\rho / \sigma)(\rho+\sigma)^{2} e^{-(\rho+\sigma) r}}{\left[1+(\rho / \sigma) e^{-(\rho+\sigma) r}\right]^{2}}, \\
& V_{4}(r)=\frac{-\rho \sigma\left\{4 \rho \sigma+(\rho-\sigma)^{2} \cosh (\rho+\sigma) r+(\rho+\sigma)^{2} \cosh [(\rho-\sigma) r-2 \theta]\right\}}{\{\sigma \cosh (\rho r-\theta)+\rho \cosh (\sigma r+\theta)\}^{2}} .
\end{aligned}
$$

In (4.10), $\alpha=-e^{-2 \theta}(\theta>0)$, in (4.11), $\alpha=0$, and in (4.12), $\alpha=e^{2 \theta}(-\infty<\theta<\infty)$. Although it has been found convenient to express the potentials in seemingly different forms, they are members of one continuous family for $-1<\alpha<\infty$ as is evident from (4.3) and (3.7). ${ }^{9}$

\footnotetext{
${ }^{9}$ The two phase equivalent potentials discussed in reference 2 are members of such a continuous family. Setting $\rho=\frac{3}{2} \lambda, \sigma=\frac{1}{2} \lambda$, $\beta=1$, one obtains (13a) for $\alpha=1$ and (13b) for $\alpha=0$.
}

By (4.6), $b_{0}=(\rho-\sigma)^{2}$, and hence, from (3.2) and (4.4),

$$
f_{2}(k)=\frac{2 k+i(\rho-\sigma)}{2 k-i(\rho+\sigma)}
$$

for $V_{2}, V_{3}$ and $V_{4}$. Consequently, these potentials are phase equivalent, and have one bound state with energy

$$
E_{2}=-\frac{1}{4}(\rho-\sigma)^{2} .
$$


At the origin, they have the common value $V(0)=-2 \rho \sigma$, but according to (4.14) they have different asymptotic forms.

Comparing now $f_{1}(k)$ with $f_{2}(k)$ we find that both families are phase equivalent (although their bound states differ) because in both cases the function $S(k)=f(k) / f(-k)$ has the value

$$
S(k)=\frac{[2 k+i(\rho-\sigma)][2 k+i(\rho+\sigma)]}{[2 k-i(\rho-\sigma)][2 k-i(\rho+\sigma)]} .
$$

(Some of the potentials are plotted in Figs. 1 and 2.)

(d) The limiting case $\sigma=0$. If $\rho$ is fixed, but $\sigma$ converges to zero, the two functions $f_{1}(k)$ and $f_{2}(k)$ converge to

$$
f_{3}(k)=(2 k+i \rho) /(2 k-i \rho),
$$

so that

$$
S_{3}(k)=[(2 k+i \rho) /(2 k-i \rho)]^{2} .
$$

The corresponding function $w_{3}(r)$ may be obtained as follows: Replace $w(r)$ in (4.3) by $w(r) / 2 \sigma$, and set either $\alpha=-1, \beta=1+(2 \sigma \tau / \rho)$, or $\alpha=(2 \sigma \tau / \rho)-1, \beta=1$, where $\tau$ is any fixed positive number. As $\sigma \rightarrow 0$, we find the function

$$
w_{3}(r)=\sinh \rho r-\rho r+\tau \quad(\tau>0)
$$

which is a solution of $Q=0$ for $c_{1}=\frac{1}{2} \rho^{2}, c_{2}=c_{1}{ }^{2}$. The associated family of phase equivalent potentials is given by

$$
V_{5}(r)=2 \rho^{2} \frac{\rho r \sinh \rho r-2(\cosh \rho r-1)-\tau}{[\sinh \rho r-\rho r+\tau]^{2}} .
$$

In the theory of the $S$-matrix the following criterion has been derived: If $k_{0}=-i \kappa_{0}\left(\kappa_{0}>0\right)$ is a zero of the function $S(k)$ which corresponds to a bound state, then $d S / d \kappa$ is negative at the point $\kappa_{0}$. Jost ${ }^{6}$ has remarked that this criterion does not necessarily hold if $f(k, r)$ is singular at $k=-k_{0}$. In fact, the potentials discussed in this section provide counter examples. At the stationary energy value $d S / d_{\kappa}$ is positive for $V_{1}$, negative for $V_{2}, V_{3}$ and $V_{4}$, and zero for $V_{5}$.

\section{RATIONAL POTENTIALS}

If $c_{1}=c_{2}=0$, the equation $R=0$ reduces to $w^{(4)}=0$. The condition $Q=0$ implies that $w(r)$ is a polynomial of first or of third degree. The first possibility may be discarded, because the associated potential has the form (2.11). The remaining solutions of $Q=0$ are given by

$$
w(r)=(r-\alpha)^{3}+\gamma^{3} .
$$

We shall assume that $\gamma>\alpha \geqq 0$. By (3.7) and (3.8)

$$
\begin{aligned}
a(r) & =-6(r-\alpha)^{2} / w(r), \\
b(r) & =-12(r-\alpha) / w(r) . \\
V_{6}(r) & =6(r-\alpha)\left[(r-\alpha)^{3}-2 \gamma^{3}\right] /(w(r))^{2} .
\end{aligned}
$$

Consequently,

$$
\begin{aligned}
& a_{\infty}=b_{\infty}=0, \quad V_{6}(r) \sim 6 / r^{2} \quad(\text { as } r \rightarrow \infty), \\
& a_{0}=-6 \alpha^{2} /\left(\gamma^{3}-\alpha^{3}\right), \quad b_{0}=12 \alpha /\left(\gamma^{3}-\alpha^{3}\right) .
\end{aligned}
$$

I. $\alpha=0$. Here, $a_{0}=b_{0}=0$, so that in view of $(5.4)$ $f(k)=1$, i.e., all phase shifts $\eta(k)$ vanish. There exists a stationary state of energy zero, since $b(r)$ (see (5.2)) is a square integrable proper function of the Schrödinger equation.

II. $\alpha>0$. Here $4 k^{2}+2 i k a_{0}+b_{0}=(2 k+i \lambda)(2 k+i \nu)$, where

$$
\left.\begin{array}{l}
\lambda \\
\nu
\end{array}\right\}=\frac{1}{2}\left\{-a_{0} \pm\left(a_{0}^{2}+4 b_{0}\right)^{\frac{1}{2}}\right\}
$$

so that $\lambda>0, \nu<0$, and $|\nu|>\lambda$. Then

$$
\begin{aligned}
f_{4}(k) & =\frac{(2 k+i \lambda)(2 k+i \nu)}{4 k^{2}} ; \\
S_{4}(k) & =\frac{(2 k+i \lambda)(2 k+i \nu)}{(2 k-i \lambda)(2 k-i \nu)}
\end{aligned}
$$

The energy of the stationary state is $E=-\frac{1}{4} \lambda^{2}$. Comparison with (2.13) shows that, for an appropriate choice of $\lambda$ and $\beta$, the Eckart potential (2.12) is phase equivalent to $V_{6}(r)$. The parameter $\lambda$ is given by (5.6), and $\beta=-\left[3 \alpha^{3} /\left(4 \gamma^{3}-\alpha^{3}\right)\right]^{\frac{1}{2}}$. (See Fig. 3.) This example is of interest because, for a negative value of $\beta$, the Eckart potential is repulsive, and has no bound state, although $V_{6}$ has the bound state $E=-\frac{1}{4} \lambda^{2}$. This is a somewhat anomalous case, due to the relatively slow decrease of $V_{6}$. As we have seen at the end of Section 1 , two phase equivalent potentials which fall off rapidly enough have the same number of bound states. A similar remark applies to the case I. Here the potential is phase equivalent to $V=0$. A more rapidly decreasing potential, however, which is phase equivalent to $V=0$ has no bound states, and consequently, it must vanish because, according to Levinson's results, two phase equivalent potentials are identical if they have no bound states.

\section{CONCLUDING REMARKS}

(a) If the function $f(k, r)$ is regular in the whole complex $k$-plane the results of the $S$-matrix theory hold without restrictions (in particular, two phase equivalent potentials have then the same stationary energies, because no "redundant" zeros occur). It can be shown that $f(k, r)$ is regular if the integrals

$$
I(\alpha)=\int_{0}^{\infty} e^{\alpha r}|V(r)| d r
$$

converge for all positive $\alpha$ (see the appendix). While, in this sense, a Gaussian potential decreases rapidly enough, this is no longer the case for exponentially decreasing potentials. It should be emphasized, however, 
that all field theories apparently lead to exponentially decreasing potentials, so that $I(\alpha)$ diverges for sufficiently large $\alpha$.

(b) So far we have restricted our discussion to the angular momentum 0 . On the basis of Levinson's results ${ }^{1}$ it can be shown that two potentials which fall off rapidly enough are dentical if they are phase equivalent for all angular momenta. Let $V_{1}$ and $V_{2}$ be two potentials, and let $\eta_{l}{ }^{\prime}(k)$ and $\eta_{l}{ }^{\prime \prime}(k)$ be the corresponding phase shifts for angular momentum $l$. If $\int_{0}^{\infty} r\left|V_{i}(r)\right| d r$ is finite $(i=1,2)$ and if for a certain $l$

$$
V_{i}(r)+l(l+1) / r^{2} \geqq 0 \quad(i=1,2)
$$

then $\eta_{l}{ }^{\prime}(k)=\eta_{l}{ }^{\prime \prime}(k)$ implies that $V_{1}(r)=V_{2}(r)$. Clearly, every potential which is bounded and which, for large $r$, is smaller than const $/ r^{2}$ satisfies the inequality (6.1) for sufficiently large $l$. Assume now that $V_{1}(r)$ and $V_{2}(r)$ are potentials of this kind, and assume that they are phase - equivalent for all $l$, i.e., that $\eta_{l}^{\prime}(k)=\eta_{l}^{\prime \prime}(k)$ for all $l$. Choose a sufficiently large $l_{0}$ so that (6.1) holds for both $V_{1}(r)$ and $V_{2}(r)$. Since $\eta l_{0}^{\prime}(k)=\eta l_{0}^{\prime \prime}(k)$ it follows that $V_{1}(r)=V_{2}(r)$. Although this argument settles the question stated in the introduction, it does not yet lead to an effective construction of a potential from its phase shifts.

In conclusion, the writer should like to thank Professor E. P. Wigner for very interesting discussions on the subject of this paper.

\section{APPENDIX}

We indicate here the proof of some assertions about the function $f(k, r)$ made in Sections 1 and 6. Set $g(k, r)=e^{i k r} f(k, r)$. Then

$$
g^{\prime \prime}-2 i k g^{\prime}=V(r) g
$$

and $\lim _{r \rightarrow \infty} g(k, r)=1$ for every real non-vanishing $k$. Following Jost, we replace (A1) by the integral equation

$$
\left\{\begin{array}{l}
g(k, r)=1+\int_{r}^{\infty} D_{k}(\rho-r) V(\rho) g(k, \rho) d \rho \\
D_{k}(\xi)=\left(1-e^{-2 i k \xi}\right) / 2 i k=\int_{0}^{\xi} e^{-2 i k \tau} d \tau
\end{array}\right.
$$

which may be solved in the familiar way by the series

$$
g(k, r)=\sum_{n=0}^{\infty} g_{n}(k, r), \quad \text { where } g_{0}(k, r)=1,
$$

and

$$
g_{n+1}(k, r)=\int_{r}^{\infty} D_{k}(\rho-r) V(\rho) g_{n}(k, \rho) d \rho
$$

provided the series converges properly.

(a) Assume that $\int_{0}^{\infty} r|V(r)| d r$ is finite, and set $X(r)$ $=\int_{r}^{\infty} \rho|V(\rho)| d \rho$. If $\operatorname{Im}(k)<0,{ }^{10}$ then, by (A2), $\left|D_{k}(\rho-r)\right| \leqq \rho$ $-r \leqq \rho$, and it follows by induction that $\left|g_{n}(k, r)\right| \leqq(X(r))^{n} / n$ ! $\leqq(\bar{X}(o))^{n} / n$ !. Consequently, the series converges to a solution. Moreover, $g(k, r)$ (and also $f(k, r)$ ) is uniformly bounded in $r$ and $k$ as long as $\operatorname{Im}(k) \leqq 0$. This holds in particular for the function $g(0, r)$ which is a solution of the Schrödinger equation for $E=0$. Since $g(0, r) \rightarrow 1$ as $r \rightarrow \infty$ it has only a finite number of zeros, and consequently the Schrödinger equation has at most a finite number of discrete proper values. A linearly independent solution of (A1) (for $k=0$ ) is given by

$$
g_{1}(r)=g(0, r) \int_{r_{0}}^{r} \frac{d \rho}{[g(0, \rho)]^{2}}
$$

for a fixed $r_{0}$. Since $\lim _{r \rightarrow \infty} g(0, r)=1$, it follows that $\lim _{r \rightarrow \infty} g_{1}(r) / r=1$. Therefore no linear combination of $g(0, r)$ and $g_{1}(r)$ is square integrable, i.e., $E=0$ is not a bound state.

(b) Let $\alpha>0$, and assume that

$$
I(\alpha)=\int_{0}^{\infty} e^{\alpha r}|V(r)| d r<\infty .
$$

Then $f(k, r)$ is regular in $k$ for $\operatorname{Im}(k)<\frac{1}{2} \alpha$.

Proof: Assume $\operatorname{Im}(k) \leqq \frac{1}{2} \alpha_{1}, \alpha_{1}<\alpha$. Observe that $\left|D_{k}(\xi)\right| \leqq \xi e^{\alpha_{1} \xi}$ $\leqq c \cdot e^{\alpha \xi}$ where $c$ is a suitably chosen constant. It follows by induction that $\left|g_{n}(k, r)\right| \leqq[c Y(r)]^{n} / n ! \leqq[c I(\alpha)]^{n} / n !$ where $Y(r)$ $=\int_{r}^{\infty} e^{\alpha \rho}|V(\rho)| d \rho$. This proves the uniform convergence of the series $\Sigma_{0}^{\infty} g_{n}(k, r)$. The regular analytic character of $g(k, r)$ (and of $f(k, r))$ is established in an analogous fashion.

Consider, for example, the potential $V$ in (2.12). There the integrals $I(\alpha)$ are finite if $\alpha<\lambda$. The associated function $f(k, r)$ is regular for $\operatorname{Im}(k)<\frac{1}{2} \lambda$, but it has a pole at $k=\frac{1}{2} i \lambda$.

If $I(\alpha)$ is finite for all $\alpha$, then $f(k, r)$ is regular in the whole complex $k$-plane.

${ }^{10} \operatorname{Im}(k)$ denotes the imaginary part of $k$. 